\title{
Parameter Estimation and Change Detection in Linear Regression Models Using Mixed Integer Linear Programming
}

\author{
Soheil Salehpour, Andreas Johansson and Thomas Gustafsson
}

\begin{abstract}
We present a method for change detection and parameter estimation in change in the mean models and $A R(X)$ model. The method is based on the assumption of piecewise constant parameters resulting in a sparse structure of their derivative. To illustrate the algorithm and the performance of it, we apply it to the change in the mean model and compare it with four other change detection algorithms. Two applications are treated with good results, fuel monitoring and airbag control. The $A R(X)$ change model, shows the good performance of this method in two illustrative examples.
\end{abstract}

Index Terms - change detection, change in the mean model, AR model, ARX model, MILP, sparsity, optimization.

\section{INTRODUCTION}

$\mathbf{T}$ HE areas of change fault detection are quite active fields, both in research and applications. Faults occur in almost all systems, and change detection has the role of locating the fault occurance in time and to give an alarm.

In [1] and [2] surveys off-line formulations of single and multiple change point estimation. In segmentation, the goal is to find a sequence $k^{n}=\left(k_{1}, k_{2}, \cdots, k_{n}\right)$ of time indices, where both the number $n$ and the locations $k_{i}$ are unknown, such that the signal can be described as piecewise constant, i.g. the change in the mean model as.

$$
y(t)=\theta(t)+e(t)
$$

where $\operatorname{Var}(e(t))=\sigma^{2}$ and if the signal model has the form of linear regression

$$
y(t)=\phi(t)^{T} \theta(t)+e(t)
$$

where $k_{i-1}<t \leq k_{i}$, and for an ARX model

$$
\begin{aligned}
\phi(t)^{T} & =\left(-y_{t-1}, \ldots,-y_{t-n_{a}}, u_{t-n_{k}}, \ldots, u_{t-n_{k}-n_{b}+1}\right) \\
\theta(t)^{T} & =\left(a_{t}^{1}, a_{t}^{2}, \ldots, a_{t}^{n_{a}}, b_{t}^{1}, b_{t}^{2}, \ldots, b_{t}^{n_{b}}\right)
\end{aligned}
$$

In change detection, $\theta(t)$ assumed piecewise constant, which gives a sparse structure of its derivative.

In Section II, a bound for the noise is defined. A MILP (Mixed Integer Linear Programming) algorithm to minimize the sparsity of a matrix is described and a method besed on this algorithm estimates the parameters. Simulation results are given in Section III. Section IV gives some concluding remarks and directions for future work.

\section{PRELIMINARIES}

The vector of dimension $n$ where each element is 1 is denoted as $1_{n}=[1,1, \ldots, 1]^{T}$ and $I_{n}$ is the identity matrix of dimension $n$.

Thomas Gustafsson, Andreas Johansson and Soheil Salehpour are with the Control Engineering Group, Luleå University of Technology, SE-971 87 Luleå, Sweden.

soheil@ltu.se, andreas.johansson@ltu.se, tgu@ltu.se

\section{A. Noise Bound}

To bound the noise $e(k)$ we will use the window norm, which for continuous time signals is defined as [6]

$$
\|e\|_{\omega}=\sup _{t \geq 0} \int_{0}^{t} \omega(t-\tau)|e(\tau)| d \tau
$$

where $\omega(t)$ is nonnegative and bounded by an exponentially decreasing function. In [5] a discrete time window norm is defined as follows

Definition 1: A window sequence is a sequence $\omega: Z^{+} \rightarrow$ $R^{+}$, which is not identically zero and satisfies $\omega(k) \leq c e^{-a k}$ for all $k \geq 0$ and some positive $c$ and $a$.

Given a window sequence $\omega(k)$, the window norm for a discrete time signal $e(k)$ may then be defined as

$$
\|e\|_{\omega}=\sup _{k \geq 0} \sum_{i=0}^{k} \omega(k-i)|e(i)|
$$

It is remarked that (3) satisfies all properties of a norm, but the proof is omitted. It is straightforward to see that the window norm is equal to the $l_{\infty}$-norm by choosing $\omega$ as the unit pulse function. The drawback of $l_{\infty}$-norm is that it only considers the peak value of the signal without any averaging and may therefore be conservative. This problem is handled in the window norm by choosing a window function that averages over a suitable time interval, i.e. a pulse function with non-unit duration or a decaying exponential function. The window norm actually approaches the $l_{1}$-norm by letting $\omega$ approach a unit step function. An assumption on the noise $e=\left[e_{1}, \ldots, e_{l}\right]$ may now be expressed as

$$
\left\|e_{j}\right\|_{\omega} \leq \epsilon_{j}, j=1, \ldots, l
$$

We assume that each $\omega(k)$ is monotone decreasing for $k>0$. Then, as showed in [5] for scalar $e$, the condition (4) can be expressed as

$$
W|E| \leq 1_{N+1} \otimes \epsilon
$$

where

$$
\begin{gathered}
W=\left[\begin{array}{cccc}
\omega(0) & 0 & \ldots & 0 \\
\omega(1) & \omega(0) & \ldots & 0 \\
\vdots & \vdots & \ddots & \vdots \\
\omega(N) & \omega(N-1) & \cdots & \omega(0)
\end{array}\right] \\
E=[e(1), \cdots, e(N)]^{T} \text { and } \epsilon=\left[\epsilon_{1}, \epsilon_{2}, \ldots, \epsilon_{l}\right]^{T} .
\end{gathered}
$$




\section{B. Sparse Matrix}

In numerical analysis, a sparse matrix is a matrix populated primarily with zeros. The concept of sparsity is useful in complex systems and many application areas such as network theory. Huge sparse matrices often appear in science or engineering when solving partial differential equations.

One common approach to seeking a sparse description is based on $l_{1}$-norm regularization [3] which produces an approximation with a sparse structure.

In this article, we represent an exact solution of maximization of sparsity by using MILP (Mixed Integer Linear Programming) to minimize the number of non-zero elements in a matrix or vector [7].

We consider the logical variable $\delta_{i j} \in\{0,1\}$ and the matrix $S=\left(s_{i j}\right)_{i, j=1 \ldots N}$ to be related as

$$
\begin{aligned}
& \delta_{i j}=1 \leftrightarrow s_{i j} \neq 0 \\
& \delta_{i j}=0 \leftrightarrow s_{i j} \equiv 0
\end{aligned}
$$

and we aim to minimize $\sum_{i, j=1}^{N} \delta_{i j}$ for $i, j=1, \ldots, N$. First, we assume $\delta_{i j}=\delta_{i j}^{1}+\delta_{i j}^{2}$ and (6) is re-written as

$$
\begin{aligned}
& \left(\delta_{i j}^{1}, \delta_{i j}^{2}\right)=(1,0) \leftrightarrow s_{i j}>0 \\
& \left(\delta_{i j}^{1}, \delta_{i j}^{2}\right)=(0,0) \leftrightarrow s_{i j}=0 \\
& \left(\delta_{i j}^{1}, \delta_{i j}^{2}\right)=(0,1) \leftrightarrow s_{i j}<0
\end{aligned}
$$

We establish a more practical link between logical and real variables by using MILP inequalities

$$
\begin{aligned}
m \delta_{i j}^{1}-M \delta_{i j}^{2} & \leq s_{i j} \leq M \delta_{i j}^{1}-m \delta_{i j}^{2} \\
\delta_{i j}^{1}+\delta_{i j}^{2} & \leq 1
\end{aligned}
$$

where $m=\min _{i, j}\left(\left|s_{i j}\right|\right)$ and $M=\max _{i, j}\left(\left|s_{i j}\right|\right)$. Then, a MILP is formulated for maximizing sparsity of $S$ as

$$
\begin{array}{cc}
\operatorname{minimize} & \sum_{i, j=1}^{N}\left(\delta_{i j}^{1}+\delta_{i j}^{2}\right) \\
\text { subject to: } & (7)
\end{array}
$$

Here, we also represent an approximation method which is more practical in a numerical sense [7]. This method instead minimizes the number of elements of a matrix which are larger than a certain threshold. If we consider $\mu$ as threshold, the logical variables are changed into

$$
\begin{aligned}
& \delta_{i j}=1 \leftrightarrow\left|s_{i j}\right|>\mu \\
& \delta_{i j}=0 \leftrightarrow\left|s_{i j}\right| \leq \mu
\end{aligned}
$$

Then, the inequalities in (7) are substituted in (8) by

$$
\begin{aligned}
2 \mu \delta_{i j}^{1}-M \delta_{i j}^{2}-\mu & \leq s_{i j} \leq M \delta_{i j}^{1}-2 \mu \delta_{i j}^{2}+\mu \\
\delta_{i j}^{1}+\delta_{i j}^{2} & \leq 1
\end{aligned}
$$

where $M=\max _{i, j}\left(\left|s_{i j}\right|\right)+\mu$ and $\mu$ is a small number.

\section{Method Based on MILP and the Derivative of Parameters}

The differentiation of a piecewise parameter produces a lot of zero samples and thus, it has a sparse structure. The timederivative of $\theta$ is approximated by $(\Delta \theta)(k)=\theta(k)-\theta(k-1)$.
Then, the following sparsity property of its derivative is used for estimating the parameters [7]. With the definitions

$$
\begin{aligned}
\sigma_{i} & =\left[\delta_{1 i}^{1}, \delta_{1 i}^{2}, \cdots, \delta_{q i}^{1}, \delta_{q i}^{2}\right] \text { for } i=1, \cdots, N, \\
\Sigma_{N} & =\left[\sigma_{0}, \sigma_{1}, \cdots, \sigma_{N-1}\right]^{T}, U=I_{N q} \otimes[M,-2 \mu], \\
L & =I_{N q} \otimes[2 \mu,-M] \text { and } \Lambda=I_{N q} \otimes[1,1]
\end{aligned}
$$

where $q=n_{a}+n_{b}$, the sparsity constraint (9) applied to the derivative $\Delta \Theta$ may be expressed as

$$
\begin{aligned}
L \Sigma_{N}-1_{N q} \mu & \leq \Delta \Theta \leq U \Sigma_{N}+1_{N q} \mu \\
\Lambda \Sigma_{N} & \leq 1_{N q}
\end{aligned}
$$

where $\Delta \Theta=\left[\Delta \theta(1)^{T} \Delta \theta(2)^{T} \cdots \Delta \theta(N)^{T}\right]^{T}$. Also, let $Y_{m}=\left[\begin{array}{lll}y_{m}(1)^{T} & y_{m}(2)^{T} \cdots & y_{m}(N)^{T}\end{array}\right]^{T}$, where $y_{m}(k)$ are the measured outputs of (1) or (2). We now use the constraint (10), and MILP-optimization to formulate

$$
\begin{array}{cc}
\underset{\Sigma_{N}, \Pi}{\operatorname{minimize}} & 1_{2 N q} \Sigma_{N}+H \epsilon \\
\text { subject to: } & (10) \\
& W\left|Y_{m}-\Xi \Theta\right| \leq \epsilon
\end{array}
$$

where $H$ is a weight for the noise, and $\Xi=I_{N q}$ in (1) and $\Xi=\operatorname{diag}\left(\phi(1)^{T}, \phi(2)^{T}, \cdots, \phi(N)^{T}\right)$ in (2).

\section{Simulation Results}

To give some idea of the performance of the MILPoptimization method, we apply it to the change mean model (1) and the change $\operatorname{AR}(X)$ model (2).

First, we consider one abrupt change of magnitude 4 in the mean signal (Fig. 1(a)), where the noise is Gaussian with variance 1 . The window function $\omega$ in the norm $\|\cdot\|_{\omega}$ is chosen as pulse function with duration time (DT) correspond to 10 . The result compares with four different change point estimations in the statistical literature [1], i.e. the Bayesian approach, the maximum likelihood approach and two nonparametric approaches. The root mean square parameter error (RMSE) for different variances of noise vs. $S / N$ is showed in Fig. 2(a) and the scaled mean square parameter error (SMSE) for the different amplitude of the second mean change vs. $S / N$ is depicted in Fig. 2(b), where the MSE and SMSE are definded as follows.

$$
\begin{aligned}
R M S E & =\frac{\sum_{t=1}^{N}\|\theta(t)-\hat{\theta}(t)\|^{2}}{N} \\
S M S E & =\frac{\sum_{t=1}^{N}\|\theta(t)-\hat{\theta}(t)\|^{2}}{\sum_{t=1}^{N}\|\theta(t)\|^{2}}
\end{aligned}
$$

The result in Fig. 2 shows that the MILP-optimization method applies less RMSE and SMSE in higher $S / N$.

In the next simulation, there are three abrupt change of magnitudes 2,3,4, respectively. The noise is Gaussian with variance 1 and $D T=5$. The result is depicted in Fig. 1(b), and the algorithm properly finds the change of mean value and estimate the magnitude of it.

Then, two illustrative applications are given here, fuel monitoring and airbag control [1]. The MILP-optimization method is applied to the fuel consumption filter problem with 


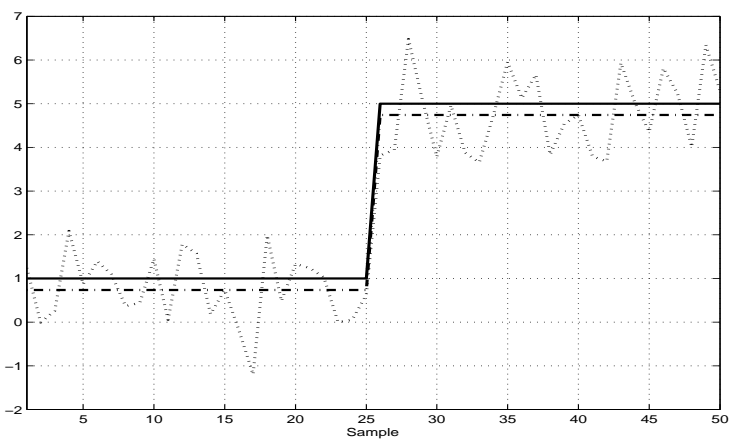

(a)

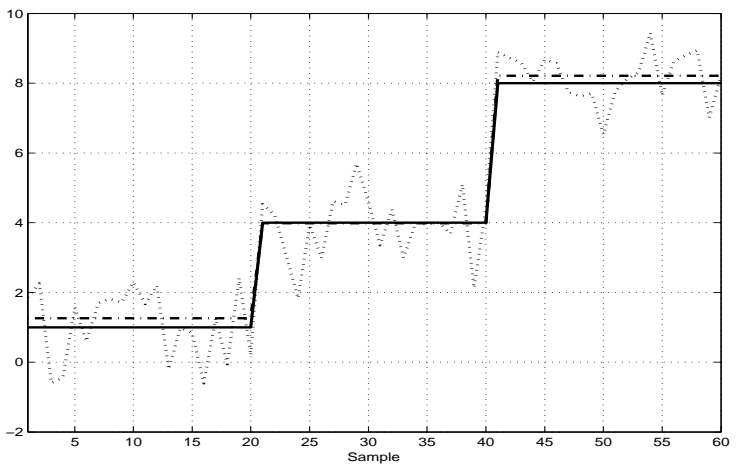

(b)

Fig. 1. The change in the mean model. The mean of signal (solid) and the mean model and Gaussian noise with $\sigma^{2}=1$ (-.) (a) An abrupt change of magnitude 4. (b) Three abrupt change of magnitudes 2,3,4.

$D T=20,5$ and to the airbag control with $D T=400,100$, which gives good detection of the change mean in both applications where the more change is detected whenever $D T$ is decreased.

In purpose to test the AR change model, we consider the AR parameters (dash and dot) in Fig. 5(a) with a Gaussian noise with variance $0.1, y(0)=1$ and $D T=5$, which the output is depicted in Fig. 5(b). The method gives a good estimation of parameters and the change of them (solid line in Fig. 5(a)).

The method is applied to ARX change model with $n_{a}=2$ and $n_{k}=n_{b}=1$, where the parameters are showed in Fig. 6(a) (dash and dot), which the output and input are depicted in Fig. 6(b), and $D T=5$. The good estimation of parameters and the change detection of them is showed in Fig. 6(a) (solid line). However, the disadvantage of this method is the long run time of the MILP-optimization.

\section{CONCLUSIONS AND FUTURE WORK}

An optimization approach is presented to estimate the mean of signal and the parameters of the $\mathrm{AR}(\mathrm{X})$ model. Two signals are used as illustraive examples and is compared with four other different point change detection. Two applications are tested here, i.e. fuel monitoring and airbag control.The method is applied to the $\operatorname{AR}(\mathrm{X})$ change model in two examples.

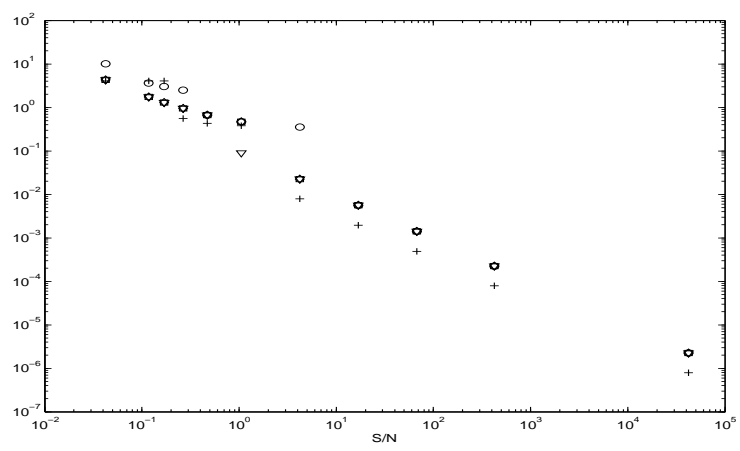

(a)

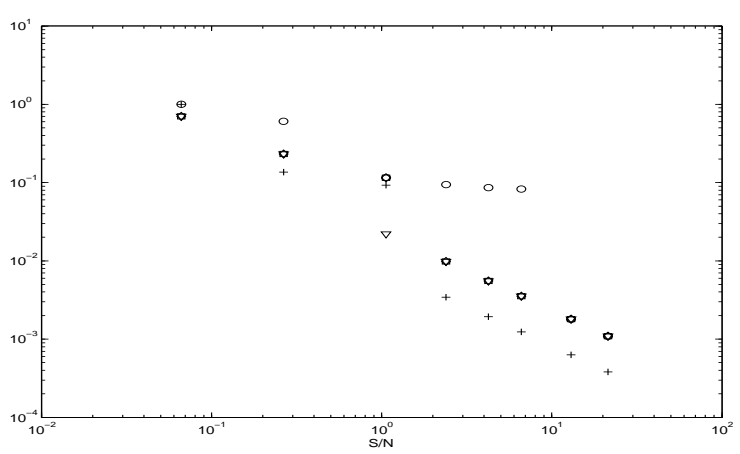

(b)

Fig. 2. (+) MILP-optimization methdo, (o) Bayesian approach, (square) ML approach, (diamond) and (triangle) are two nonparametric approaches

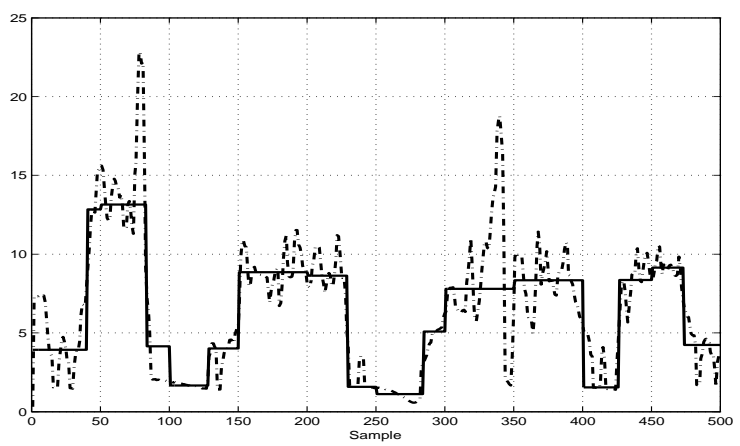

(a)

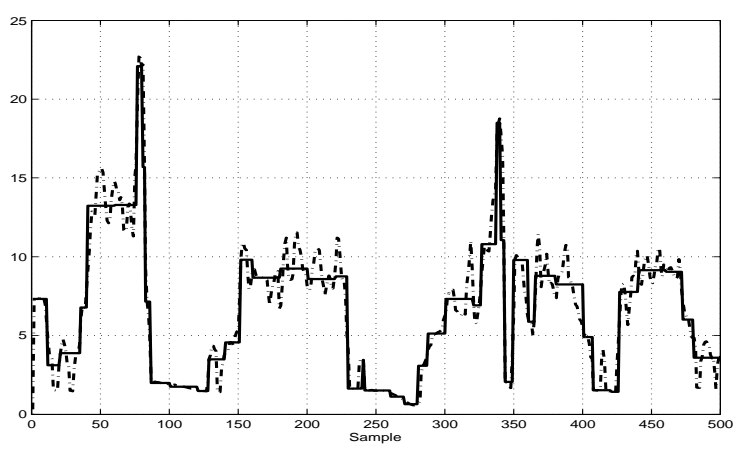

(b)

Fig. 3. The fuel consumption mesurement data (-.), the estimate mean signal (solid). (a) $D T=5$ (b) $D T=20$ 


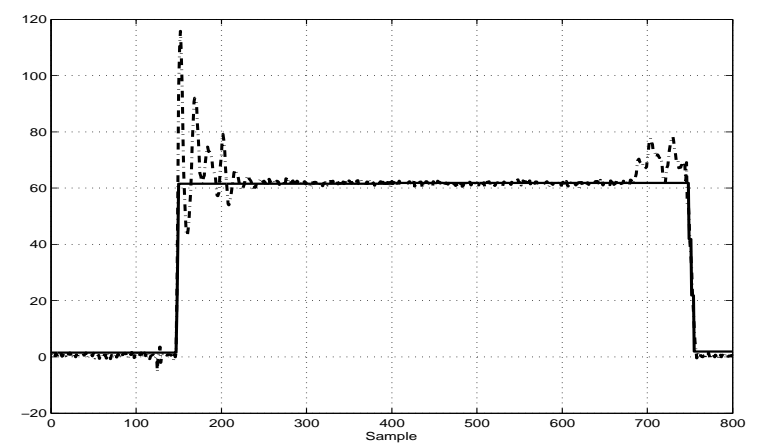

(a)

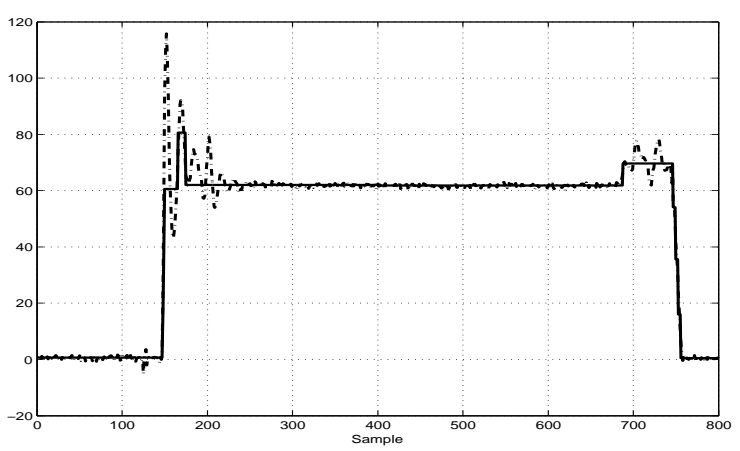

(b)

Fig. 4. The airbag control mesurement data (-.), the estimate mean signal (solid). (a) $D T=100$ (b) $D T=400$

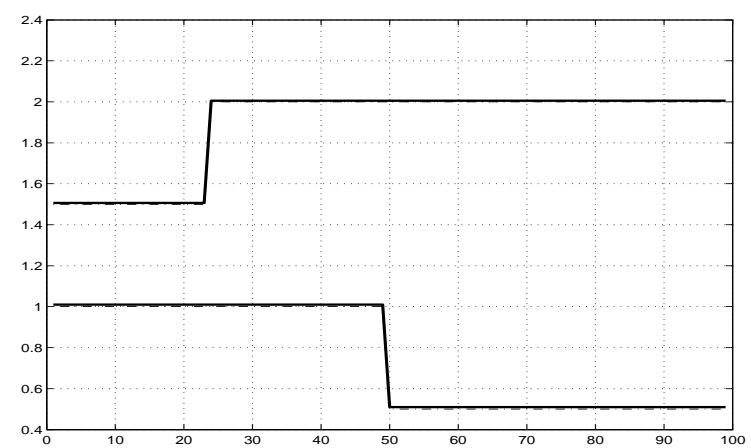

(a)

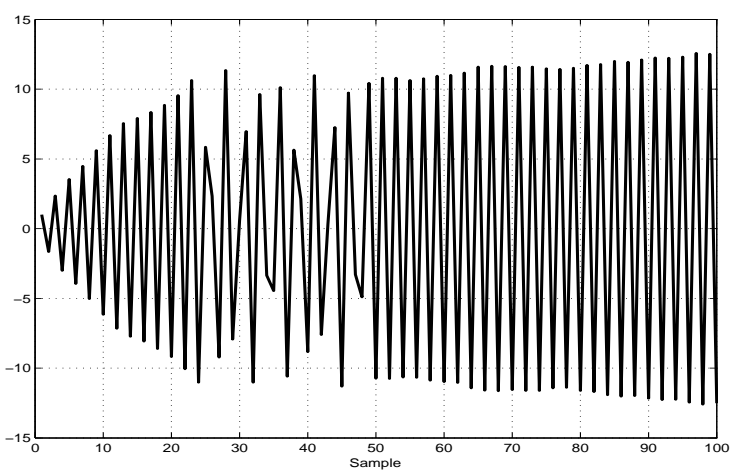

(b)

Fig. 5. The AR change model with a Gaussian noise $\left(\sigma^{2}=1\right)$. (a) The estimate parameters (solid) and parameters(-.) (b) The output

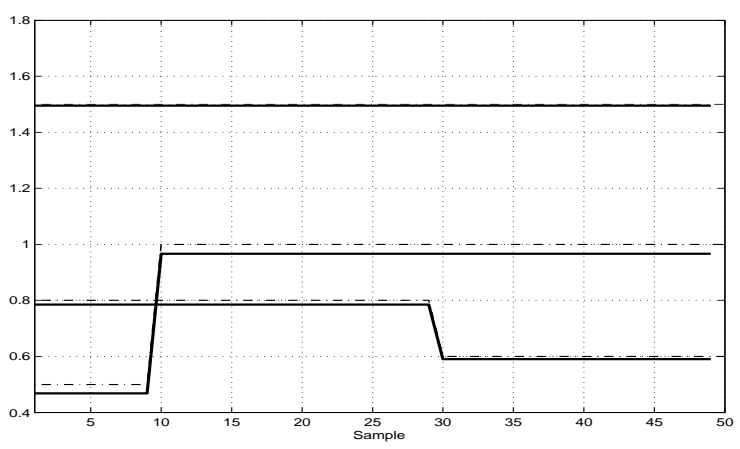

(a)

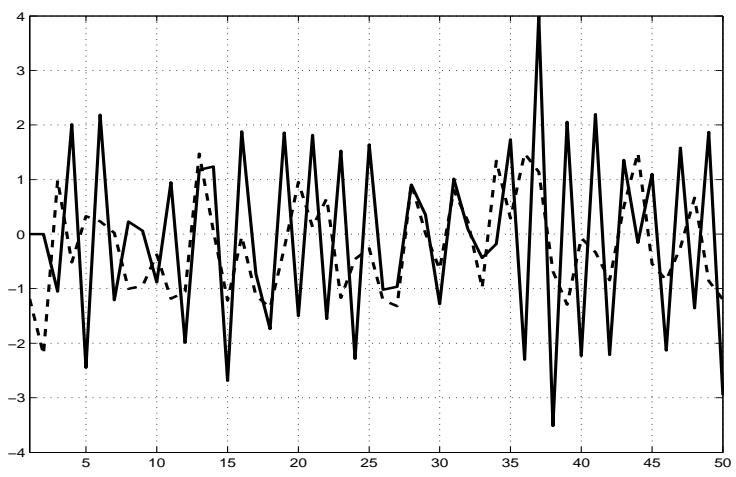

(b)

Fig. 6. The ARX change model with a Gaussian noise $\left(\sigma^{2}=1\right)$. (a) The estimate parameters (solid) and parameters(-.) (b) The output (solid) and input (-.)

For future research, other properties of noise and perturbations may also be exploited, e.g. that the noise is expected to be random and uncorrelated with the perturbations.

\section{ACKNOWLEDGMENTS}

The authors wish to thank Hjalmar Lundbohm Research Center (HLRC) funded by LKAB for financing this research.

\section{REFERENCES}

[1] F. Gustafsson. Adaptive filtering and change detection. John Wiley and Sons, Ltd, 2000

[2] S. M. Kay. Fundamentals of Statistical Signal Processing: Detection Theory. Prentice-Hall, 1998.

[3] S. Boyd and L. Vandenberghe. Convex Optimization. Cambridge University, 2007 Stephen Boyd (with Lieven Vandenberghe, 2004)

[4] J. Löfberg. YALMIP : A Toolbox for Modeling and Optimization in MATLAB. In Proceedings of the CACSD Conference, Taipei, Taiwan, 2004

[5] A. Johansson. Validation of state-space models with time-varying parameter uncertainty. In Proceedings of the $46^{\text {th }}$ IEEE Conference on Decision and Control, New Orleans, USA, 2007.

[6] A. Johansson. Shift-invariant signal norms for fault detection and control. System and Control letters, 57, 105-111, 2008.

[7] S. Salehpour and A. Johansson. Two Algorithms for Model Quality Estimation in State-Space Systems with Time-Varying Parameter Uncertainty, Accepted in ACC, 2008. 